\title{
A NOTE ON SOLUTIONS OF SCHIFFER'S DIFFERENTIAL EQUATION
}

\author{
RONALD J. LEACH \\ Department of Mathematics \\ Howard University \\ Washington, D.C. 20059 \\ (Recelved on February 4, 1982 and in revised form August 20, 1982)
}

ABSTRACT. We consider Schiffer's differential equation for functions in the class of normalized univalent functions which maximize the th coefficient. By considering a class of functionals converging to the $n$th coefficient functional, we determine some additional symetries that extrenal functions possess.

KEY WORDS AND PHRASES. Univalent function, Schiffer's differential equation, variat1on, Marty relation.

1980 NUS SUBJECT CLASSIFICATION: 30C50, 30C70, $30 C 75$.

\section{IMTRODUCTION.}

Let $S$ denote the class of functions $f(z)=z+a_{2} z^{2}+\ldots$, analytic and univalent In the unit disc D. S so well-known to be compact in the topology of uniform convergence on compact subsets of $D$. Therefore, variational problems of the form $\operatorname{Re} \phi(f)$ max mat have colutions in $S$ whenever $\phi$ 1s a continuous functional on $S$. By constructing univalent variations, Schiffer $[1,2]$ showed that an extremal function $f$ for $\phi(f)$ - Re $a_{n}$ mat satisfy the differential equation

$$
\left(\frac{\zeta f^{\prime}(\zeta)}{f(\zeta)}\right)^{2} P(f(\zeta))=q(\zeta) \quad \zeta \in D
$$

where $p$ and $q$ are rational functions and $q\left(e^{1 \theta}\right)>0$. The coefficients of $p$ and $q$ depend on the function $f$ and therefore (1.1) 1s a functional-differential equation.

Por examples of the uses of the variational method, see [1-10]. For consistency, we follow the notation in Powerenke $[8, p, 183-190]$. It 1s shown there that $f$ is a solution to the problew of maximizing Re $a_{n}$; then

$$
p(f(\zeta))=\frac{1}{n} \phi_{n}^{\prime}\left(\frac{1}{f(\zeta)}\right)-a_{n} \text {. }
$$




$$
q(\zeta)=(n-1) a_{n}+\sum_{j=1}^{n-1}\left(j a_{j} \zeta^{-(n-j)}+j \bar{a}_{j} \zeta^{n-j}\right) .
$$

Here $\phi_{n}\left(\frac{1}{f(\zeta)}\right)$ is the familiar Faber polynomial of degree $n$ for $\frac{1}{f(\zeta)}$. Equation (1.1) with $\mathrm{p}$ and $\mathrm{q}$ defined by (1.2) and (1.3) respectively is the Schiffer differential equation which any function maximizing $R e a_{n}$ must satisfy. (Unfortunately, there are other solutions that are not extremal functions such as $z\left(1-z^{2}\right)^{-1}$ in the case $n=3$.)

In this note, we consider a class of functionals $T_{r}(f)$ that converge to $\Phi(f)=$ Re $a_{n}$ as $r \rightarrow 0$. We compute the Schiffer differential equation for each of these functionals and obtain new conditions that the extremal functions must satisfy. In certain cases, we show that the extremal function must satisfy an infinite system of differential equations. The equations in this system are of the form (1.1) and have the unknown coefficients of the extremal function appear in the equation.

2. THE MAIN THEOREM.

We will need the following result whose proof is an immediate consequence of the formula for the sum of a geometric progression.

Lemma. Let $g(z)=\sum_{n=0}^{\infty} b_{n} z^{n}$. Then $\sum_{i=0}^{n-1} g\left(r e^{\frac{2 \pi i j}{n}}\right)=n \sum_{i=0}^{\infty} b_{k n} r^{k n}$.

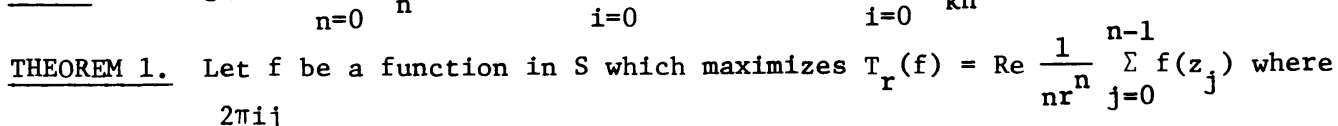
where $z_{j}=r e^{\frac{2 \pi i j}{n}}, r>0$. Then

1) with the notation $B_{j}=f\left(z_{j}\right)$, f must satisfy

$\left(\frac{z f^{\prime}(z)}{f(z)}\right)^{2} \sum_{j=0}^{n-1} \frac{B_{j}^{2}}{f(z)-B_{j}}=\frac{1}{2} \sum_{j=0}^{n-1} z_{j} f^{\prime}\left(z_{j}\right) \frac{z+z j}{z-z_{j}}+\frac{1}{2} \sum_{j=0}^{n-1} \overline{z_{j}} \overline{f^{\prime}\left(z_{j}\right)} \frac{1+z_{j} z}{1-\bar{z} z}-\operatorname{Re} \sum_{j=0}^{n-1} f\left(z_{j}\right)$

ii) as $r \rightarrow 0$, the functions $f$ (which may depend on $r$ ) approach a function in $S$ which maximizes $\operatorname{Re} a_{n}$.

PROOF. We follow the outline in Pommerenke [8, p. 183-190]. $T_{r}$ is a linear functional of degree $\mathrm{n}$ and consequently

where

$$
\left(\frac{\zeta f^{\prime}(\zeta)}{f(\zeta)}\right)^{2} p(f(\zeta))=q(\zeta)
$$

$$
p(w)=\sum_{j=0}^{n-1} \frac{B_{j}^{2}}{w-B_{j}}
$$

and 


$$
q(\zeta)=\frac{1}{2} \sum_{j=0}^{n-1} z_{j} f^{\prime}\left(z_{j}\right) \frac{\zeta+z_{j}}{\zeta-z_{j}}+\frac{1}{2} \sum_{j=0}^{n-1} z_{j} f^{\prime}\left(z_{j}\right) \frac{1+\bar{\zeta} z_{j}}{1-\bar{\zeta} z_{j}}-\operatorname{Re} \sum_{j=0}^{n-1} f\left(z_{j}\right) .
$$

Th: rroves the first statement of the theorem if we replace $\zeta$ by $z$. To prove the si d statement, note that the lemma implies

and hence

$$
\sum_{j=0}^{n-1} f\left(e^{\frac{2 \pi i j}{n}}\right)_{r}=n a_{n} r^{n}+n a_{2 n} r^{2 n}+\ldots
$$

$$
T_{r}(f)=a_{n}+0\left(r^{n}\right) .
$$

If $\varepsilon>0$ is given, we may choose $r$ so that $0\left(r^{n}\right)<\varepsilon$. Then any function $f_{0}$ maximizing $\operatorname{Re} a_{n}$ has

$$
\operatorname{Re} T_{t}(f)<\operatorname{Re} a_{n}+\varepsilon
$$

and hence $f_{0}$ is the limit of functions maximizing $\operatorname{Re}_{\mathrm{r}_{\mathrm{f}}}$ as $\mathrm{r} \rightarrow 0$.

REMARKS. 1. It is well-known that a function that maximizes Re $a_{n}$ actually has $a_{n}>0$.

2. If $f(z)=z+a_{2} z^{2}+\ldots+a_{n} z^{n}+\ldots$ maximizes Re $a_{n}$, then so do the functions

$$
e^{-\frac{2 \pi k j}{n-1}}+\left(e^{\frac{2 \pi i j}{n-1}} z\right)=z+e^{\frac{2 \pi i j}{n-1}} a_{2} z^{2}+\ldots+a_{n} z^{n}+\ldots .
$$

Our technique of approxımating $\operatorname{Re} a_{n}$ by $\operatorname{Re} T_{r}(f)$ will yield only one of the rotations of $f$; the others can be sbtained by considering replacing $r$ by $r_{j}=e^{\frac{2 \pi 1 j}{n-1}} r$. This observation will explain some later results. COROLLARY 1. There is a function $f \varepsilon S$ which maximizes Re $a_{n}$ for which

$$
2 a_{2} a_{n}=(n+1) a_{n+1}-(n-1) \overline{a_{n-1}} \text {. }
$$

PROOF. Let $F(z)$ denote the expression appearing in (2.1). We fix $r>0$ and expand both representations for $F(z)$ about $z=0$ obtaining, since $f(z)=z+a_{2} z^{2}+\ldots$,

$$
\begin{aligned}
-\sum_{j=0}^{n-1} B_{j} & +\left(-n-2 a_{2} \sum_{j=0}^{n-1} B_{j}\right) z+0\left(z^{2}\right)=-\frac{1}{2} \sum_{j=0}^{n-1}\left[z_{j} f^{\prime}\left(z_{j}\right) \frac{1+z / z_{j}}{1-z / z_{j}}\right]+ \\
& +\frac{1}{2} \sum_{j=0}^{n-1} \overline{z_{j}} \overline{f^{\prime}\left(z_{j}\right)} \frac{1+\overline{z_{j} z}}{1-\overline{z_{j} z}}-\operatorname{Re}_{j=0}^{n-1} B_{j}=-\frac{1}{2} \sum_{j=0}^{n-1}\left[z_{j} f^{\prime}\left(z_{j}\right)\left(1+\frac{2 z}{z_{j}}+0\left(z^{2}\right)\right)\right] \\
& +\frac{1}{2} \sum_{j=0}^{n-1}\left[\overline{z_{j}} \overline{f^{\prime}\left(z_{j}\right)}\left(1+2 \overline{z_{j} z}+0\left(z^{2}\right)\right]-\operatorname{Re} \sum_{j=0}^{n-1} f\left(z_{j}\right)=-i I m \sum_{j=0}^{n-1} z_{j} f^{\prime}\left(z_{j}\right)\right.
\end{aligned}
$$




$$
-\operatorname{Re} \sum_{j=0}^{n-1} f\left(z_{j}\right)+\left(\sum_{j=0}^{n-1}\left[-f^{\prime}\left(z_{j}\right)+\bar{z}_{j}^{2} f^{\prime}\left(z_{j}\right)\right]\right) z+0\left(z^{2}\right) .
$$

Equating coefficients, since $B_{j}=f\left(z_{j}\right)$, we obtain

$$
\begin{aligned}
-\sum_{j=0}^{n-1} f\left(z_{j}\right) & =-i \operatorname{Im} \sum_{j=0}^{n-1} z_{j} f^{\prime}\left(z_{j}\right)-\operatorname{Re} \sum_{j=0}^{n-1} f\left(z_{j}\right) \\
-n-2 a_{2} \sum_{j=0}^{n-1} f\left(z_{j}\right) & =-\sum_{j=0}^{n-1} f^{\prime}\left(z_{j}\right)+\sum_{j=0}^{n-1} \bar{z}_{j}^{2} \frac{f^{\prime}\left(z_{j}\right)}{}
\end{aligned}
$$

Applying the argument of the lemma to (2.2) and (2.3), we obtain

$$
\begin{aligned}
& -n a_{n} r^{n}+0\left(r^{2 n}\right)=-i I m n(n+1) a_{n+1} r^{n}-\operatorname{Re} n a_{n} r^{n}+0\left(r^{2 n}\right) \\
& -n-2 a_{2} a_{n} r^{n}+0\left(r^{2 n}\right)=-n-n(n+1) a_{n+1} r^{n}+n(n-1) \bar{a}-1+r^{n}+0\left(r^{2 n}\right) \text {. }
\end{aligned}
$$

Upon dividing (2.5) by $\mathrm{r}^{\mathrm{n}}$ and letting $\mathrm{r} \rightarrow 0$, we obtain

$$
2 a_{2} a_{n}=(n+1) a_{n+1}-(n-1) \overline{a_{n-1}} .
$$

REMARK. The conclusion of the corollary is the well-known Marty relation. It was originally derived by very elementary methods. Hummel [6, p. 77] observed that the Marty relation can also be obtained by considering the Schiffer differential equation for the functional Re $a_{n}$.

3. CONSEQUENCE OF THE MAIN THEOREM.

THEOREM 2. Suppose that a fixed function $f$ maximizes $R e T_{r}(f)$ for some sequence of $r$ 's converging to 0 . Then $f$ satisfies the system of functional-differential equations

$$
\begin{aligned}
& \left(\frac{z f^{\prime}(z)}{f(z)}\right)^{2} \frac{1}{k n} \phi_{k n}^{\prime}\left(\frac{1}{f(z)}\right)-a_{n}=(k n-1) a_{k n}+ \\
& \underset{n=1}{k n-1}\left(j a_{j} z^{-(k n-j)}+j \bar{a}_{j} z^{k n-j}\right), k=1,2, \ldots
\end{aligned}
$$

where $\phi_{k n}(w)$ is the knth Faber polynomial for W.

PROOF. By Theorem 1, f must satisfy the functional-differential equation

$$
\begin{aligned}
\left(\frac{z f^{\prime}(z)}{f(z)}\right)^{2} \sum_{j=0}^{n-1} \frac{B_{j}^{2}}{f(z)-B_{j}} & =\frac{1}{2} \sum_{j=0}^{n-1} z_{j} f^{\prime}\left(z_{j}\right) \frac{z+z_{j}}{z-z_{j}}+ \\
& +\frac{1}{2} \sum_{j=0}^{n-1} \overline{z_{j}} \overline{f^{\prime}\left(z_{j}\right)} \frac{1+\overline{z_{j} z}}{1-\overline{z_{j} z}}-\operatorname{Re} \sum_{j=0}^{n-1} f\left(z_{j}\right)
\end{aligned}
$$

where $B_{j}=f\left(z_{j}\right)=f\left(r e^{\frac{2 \pi i j}{n}}\right)$. For fixed $z$, the expression $F(z)$ defined by 
is an analytic function of $r$ for $r$ in some small interval about 0 . We expand (3.2) in powers of $r$ noting that the lemma insures that only powers of $r^{\mathrm{kn}}$ can appear. We show the argument only for powers of $\mathbf{r}^{n}$ since the computation for higher powers is similar.

$$
\begin{aligned}
& \left(\frac{z f^{\prime}(z)}{f(z)}\right)^{2} \sum_{j=0}^{n-1}\left[\frac{B_{j}^{2}}{f(z)}\left(1+\frac{B_{j}}{f(z)}+\frac{B_{j}^{2}}{f(z)^{2}}+\ldots\right)\right] \\
& =\frac{1}{2} \sum_{j=0}^{n-1} z_{j} f^{\prime}\left(z_{j}\right)\left(1+\frac{2 z j}{z}+\ldots\right) \\
& +\frac{1}{2} \sum_{j=0}^{n-1} \overline{z_{j}} f^{\prime}\left(z_{j}\right)\left(1+2 \overline{z_{j}}{ }^{2}+\ldots\right)-\operatorname{Re} \sum_{j=0}^{n-1} f\left(z_{j}\right) . \\
& \left(\frac{z f^{\prime}(z)}{f(z)}\right)^{2}\left[n r^{n}\left(\frac{C_{1}(n)}{f(z)}+\frac{C_{2}(n)}{f(z)^{2}}+\ldots+\frac{1}{f(z)^{n-1}}\right)+0\left(r^{2 n}\right)\right] \\
& =\frac{1}{2} n^{2} a_{n} r^{n}+0\left(r^{2 n}\right)+\sum_{j=0}^{n-1}\left[z_{j}^{2} \frac{f^{\prime}\left(z_{j}\right)}{z}+z_{j}^{2} \frac{f^{\prime}\left(z_{j}\right)}{z^{2}}+\ldots\right]+\frac{1}{2} n^{2} a_{n} n^{n} \\
& +0\left(r^{2 n}\right)+\sum_{j=0}^{n-1}\left[\bar{z}_{j}^{2} \overline{f^{\prime}\left(z_{j}\right) z}+\bar{z}_{j}^{3} \overline{f^{\prime}\left(z_{j}\right)} z^{2}+\ldots\right]-\operatorname{Re} n a_{n} r^{n}+0\left(r^{2 n}\right) \\
& =\left[\left(n^{2}-n\right) a_{n}+n \sum_{j=1}^{n-1}\left(j a_{j} z^{-(n-j)}+j \bar{a}_{j} z^{n-j}\right)\right] r^{n}+0\left(r^{2 n}\right) \text {. }
\end{aligned}
$$

The coefficients $C_{m}(n)$ are obtained in the following manner:

$$
\begin{aligned}
\mathrm{nr}^{n} C_{1}(n) & =\sum_{j=0}^{n-1} B_{j}^{2} \\
& =\sum_{j=0}^{n-1}\left(f\left(z_{j}\right)\right)^{2} \\
& =\sum_{j=0}^{n-1}\left(z_{j}+a_{2} z_{j}^{2}+\ldots\right)^{2} \\
& =\sum_{j=0}^{n-1} \sum_{m=1}^{\infty} \sum_{1}+m_{2}=m^{a_{m_{1}} a_{2}}\left(r e^{\frac{2 \pi 1 j}{n}}\right)^{m} \\
& =n r^{n} \sum_{m_{1}+m_{2}=n} a_{m_{1}} a_{m_{2}}+0\left(r^{2 n}\right) .
\end{aligned}
$$




$$
\begin{aligned}
& n r^{n} C_{2}(n)=\sum_{j=0}^{n-1} B_{j}^{3} \\
& =\sum_{j=0}^{n-1}\left(z_{j}+a_{2} z_{j}^{2}+\ldots\right)^{3} \\
& =\sum_{j=0}^{n-1} \sum_{m=1}^{\infty} \sum_{a_{m_{1}}}{ }^{a} m_{2}{ }^{a} m_{3}\left(r e^{\frac{2 \pi 1 j}{n}}\right)^{m} \\
& =n r^{n} \sum a_{m_{1}} a_{m_{2}}{ }^{a} m_{3}+o\left(r^{2 n}\right) .
\end{aligned}
$$

The sum $\sum_{a_{m_{1}}}{ }^{a}{ }_{2}{ }^{a} m_{3}$ is taken over all positive integers $m_{1}, m_{2}, m_{3}$ with $\mathrm{m}_{1}+\mathrm{m}_{2}+\mathrm{m}_{3}=\mathrm{n}$. This procedure yields in general

$$
\mathrm{nr}^{\mathrm{n}} \mathrm{C}_{\ell}(\mathrm{n})=\mathrm{nr} \mathrm{n}^{\mathrm{n}} \mathrm{a}_{\mathrm{m}_{1}} \ldots \mathrm{a}_{\mathrm{m}_{\ell+1}}
$$

where $m_{1}+m_{2}+\ldots+m_{\ell}=n, m_{1}>0$. We recognize $\Sigma_{\ell=1}^{n-1} c_{\ell}(n) f^{-\ell}$ as $\frac{1}{n} \phi_{n}^{\prime}\left(\frac{1}{f(z)}\right)-a_{n}$ $[8, p .57]$. This proves the result if $k=1$. The other equations for $k=2, \ldots$ are obtained in a similar manner by equating coefficients of higher powers of $r^{n}$. REMARKS. 1. A result of Pfluger [7] shows that a Koebe function $k(z)=z\left(1-e^{i \theta} z\right)^{-2}$ always satisfies (1.1).

2. The assumption that $f$ is essentially the only extremal function for the problem of maximizing $R e a_{n}$ is used quite strongly in this proof. If there were more than one function, the coefficients of the extremal function for $T_{r}$ would depend upon $r$, making the functional-differential equations even more complicated. It seems reasonable to suppose that there is essentially one extremal function (apart from rotations) for each $\mathrm{n}$ but we are unable to prove this.

3. The equation for $k=1$ is of course the familiar Schiffer differential equation for a function $f$ maximizing $R e a_{n}$. The nature of this family of equations suggests that, if $f(z)=z+a_{2} z^{2}+\ldots$ is a function which maximizes $\operatorname{Re} a_{n}, f$ also maximizes $\operatorname{Re} a_{2 n}, \operatorname{Re} a_{3 n}, \ldots$. If so, the Bieberbach conjecture would follow from a result of Hayman [11, p. 104]. He showed that, if $f \in S$, $\lim _{k \rightarrow \infty} \frac{\left|{ }^{a} k\right|}{k}=\alpha \leq 1$, with $\alpha=1$ only if $f(z)=z\left(1-e^{i \theta} z\right)^{-2}$. THEOREM 3. Suppose that $f$ satisfies the hypothesis of Theorem 2. Then f satisfies the functional equation 


$$
\frac{\frac{1}{k n} \phi_{k n}^{\prime}\left(\frac{1}{f(z)}\right)-a_{k n}}{\frac{1}{l n} \phi_{\ell n}^{\prime}\left(\frac{1}{f(z)}\right)-a_{\ell n}}=\frac{(k n-1) a_{k n}+\sum_{n=1}^{k n-1}\left(j a_{j} z^{-(k n-j)}+j a_{j} z^{k n-j}\right)}{(\ln -1) a_{\ell n}+\sum_{j=1}^{n-1}\left(j a_{j} z^{-(l n-j)}+j a_{j} z^{\ell n-j}\right)}
$$

and hence $\mathrm{f}$ is algebraic.

PROOF. Divide the kth equation in the system (3.1) by the lth equation.

The following result is of interest only if the Bieberbach.conjecture is false. THEOREM 4. Suppose there is a function $f$ not of the form $z\left(1-e^{i \theta} z\right)^{-2}$ and that $f$ satisfies the hypothesis of Theorem 2. Then there is a number $\theta_{0}$ such that e is simultaneously a zero of

$$
a_{k}(z)=(k n-1) a_{n k}+\sum_{j=0}^{k n-1}\left(j a_{j} e^{-(k n-j) 1 \theta}+j a_{j} e^{(k n-j) i \theta}\right) k=1,2, \ldots
$$

PROOF. Pfluger [7] has shown that if $f$ is a function that maximizes Re $a_{n}$, then $\operatorname{Re}\left[\frac{1}{n}{ }_{n}^{\prime}\left(\frac{1}{f(z)}\right)-a_{n}\right]<0$ unless $f$ is a rotation of the Koebe function. (He actually proved this theorem for any linear functional and the rational function $p$ related to it by (1.2).) It is well-known that $\frac{1}{n} \phi_{n}^{\prime}\left(\frac{1}{f(z)}\right)-a_{n}=0$ if and only if $f$ is a Koebe function. (See [8, p. 194], [6, Theorem 13.6].)

We consider equation (3.5) with $\ell=1$. It is well-known that since $f$ maximizes Re $a_{n}$, the function $q$ defined by (1.3) must have at least one zero on $z=1$. Since the left-handed side of (3.5) is analytic by assumption, each zero $e^{1 \theta}$ of

$$
q_{1}\left(e^{1 \theta}\right)=(n-1) a_{n}+\sum_{j=1}^{n-1}\left(j a_{j} e^{-(n-j) i \theta}+j a_{j} e^{-(n-j) 1 \theta}\right.
$$

must also be a zero of $q_{k}\left(e^{i \theta}\right), k=1,2, \ldots$. This completes the proof.

THEOREM 5. Suppose that $\mathrm{f}$ satisfies the hypothesis of Theorem 2. Then

$$
\begin{aligned}
& \text { 1) } a_{k n} \text { is real } k=1,2, \ldots \\
& \text { ii) } 2 a_{2} a_{k n}=(k n-1) a_{k n+1}-(k n-1) \overline{a_{k n-1}} \text {. }
\end{aligned}
$$

PROOF. Since $f$ is essentially the unique function maximizing $\operatorname{Re} a_{n}$, the equations (2.2) and (2.3) are valid for all $r=\left|z_{j}\right|$ in some neighborhood of 0 . Equating coefficients of $\mathrm{r}^{\mathrm{kn}}$ in (2.2) yields, after an application of the lemma,

$$
-n a_{k n}=\text { in Im kn } a_{k n}-n \text { Re } a_{k n}
$$

or

$$
-1 \text { Im } a_{k n}=-i \text { Im } k n a_{k n}
$$


which implies that $a_{k n}$ is real.

Equating coefficients in (1.6) and applying the lemma, we obtain

$$
-2 a_{2} n a_{k n}=-n(k n+1) a_{k n+1}+n(n k-1) \overline{a_{n k-1}}
$$

and the result follows after division by $-n$.

ACKNOWLEDGEMENTS. I would like to thank Professors J.A. Hummel and W.E. Kirwan for helpful conversations regarding the extremal functions for $T_{r}(f)$. I would also like to thank Professor A. Pfluger for pointing out an error in an earlier version of this paper.

\section{REFERENCES}

1. SCHIFFER, M. "A Method of Variation Within the Family of simple Functions", Proc. London Math. Soc. 44 (1938), 432-449.

2. SCHIFFER, M. "On the coefficients of Simple Functions", Proc. London Math. Soc. 44 (1938), 450-452.

3. AHLFORS, L. "Conformal Invariants", McGraw-Hi11, New York, 1973.

4. BRICKMAN, L., and WILKEN, D. "Support Points of the set of Univalent Functions", Proc. Amer. Math. Soc. 42 (1974), 523-528.

5. GARABEDIAN, P. and SCHIFFER, M. "A Coefficient Inequality for Schlicht Functions", Ann. of Math. 61 (1955), 116-136.

6. HUMMEL, J.A. "Lectures on Variational Methods in the Theory of Univalent Functions", Univ. of Maryland Lecture Notes, 1970.

7. PFLUGER, A. "Lineare Extremal probleme bei schlicten Funktionen", Ann. Acad. Sc1. Fenn. A1 489 (1971).

8. POMMERENKE, CHR. "Univalent Functions", Vandenhoeck and Ruprecht, Gottingen, 1975 .

9. SCHAEFFER, A.C., and SPENCER, D.C. "Coefficient Regions for sch1icht Functions", Amer. Math. Soc. Colloq. Pub1. vo1. 35, New York, 1950.

10. SCHOBER, G. "Univalent Functions - Selected Topics", Springer-Verlag, Berlin, 1974.

11. HAYMAN, W.K. "Multivalent Functions", Cambridge University Press, Cambridge, 1958. 


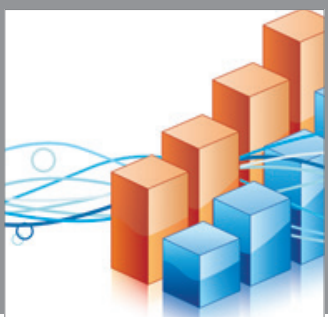

Advances in

Operations Research

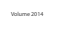

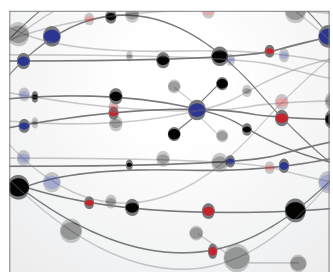

\section{The Scientific} World Journal
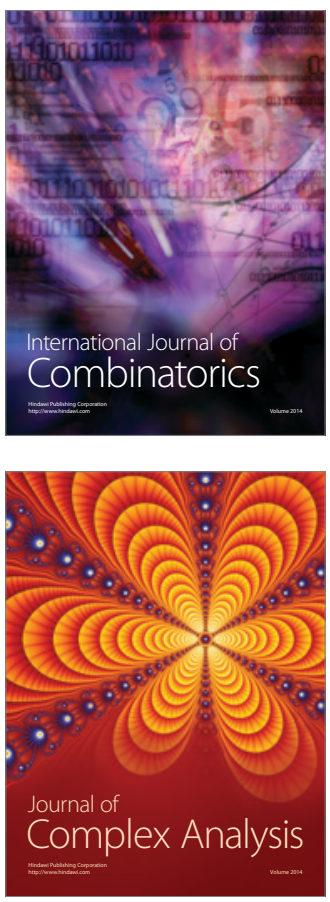

International Journal of

Mathematics and

Mathematical

Sciences
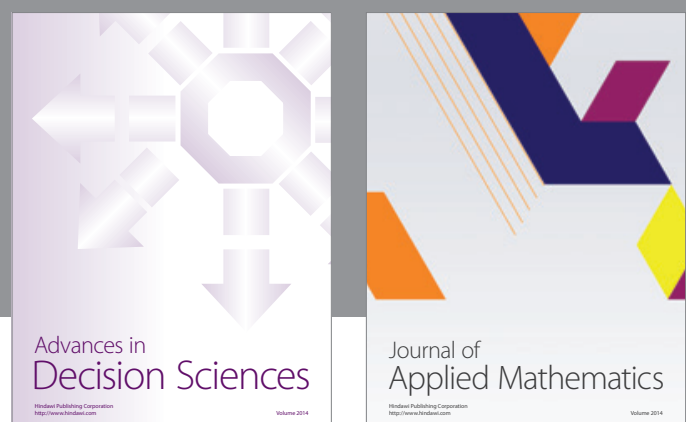

Journal of

Applied Mathematics
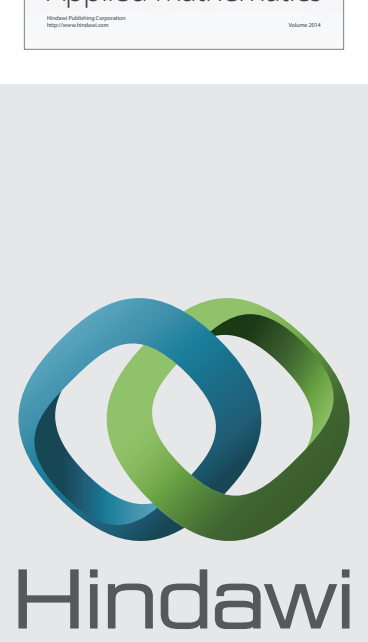

Submit your manuscripts at http://www.hindawi.com
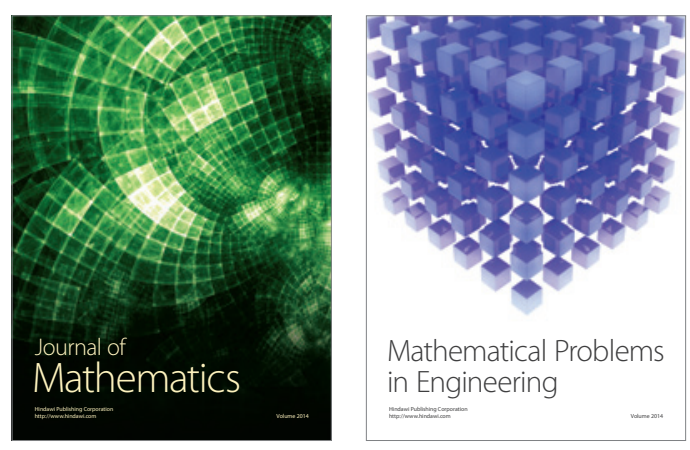

Mathematical Problems in Engineering
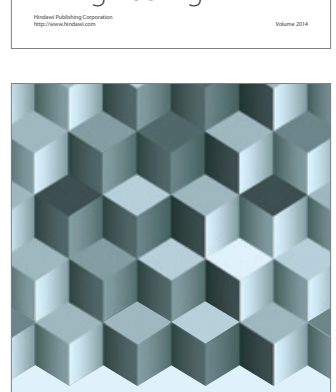

Journal of

Function Spaces
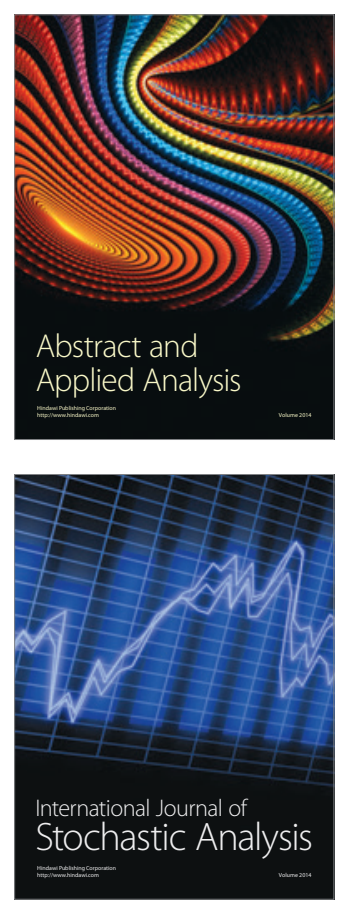

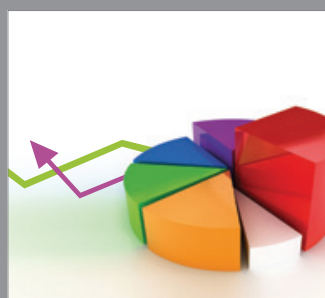

ournal of

Probability and Statistics

Promensencen
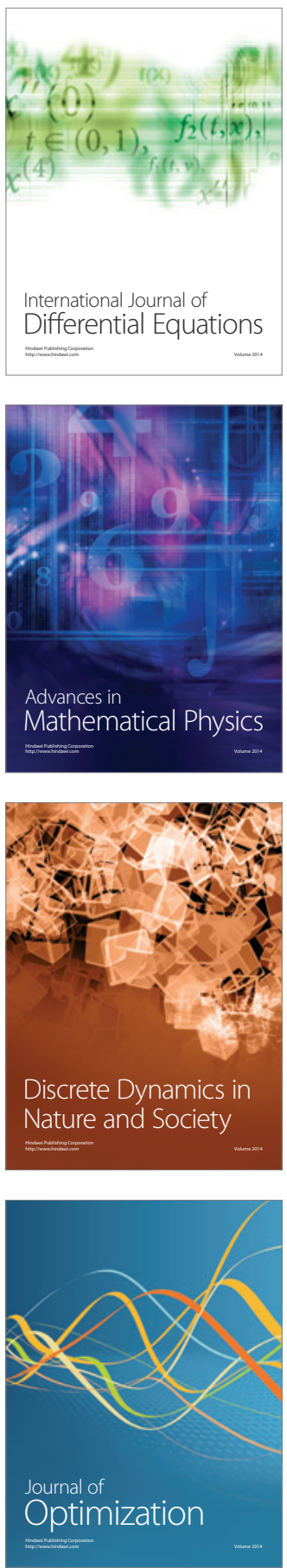